\title{
Quality assurance of malaria rapid diagnostic tests used for routine patient care in rural Tanzania: microscopy versus real-time polymerase chain reaction
}

Irene M Masanja ${ }^{1 *}$, Meredith L McMorrow ${ }^{2,3}$, Mussa B Maganga', Debora Sumari ${ }^{1}$, Venkatachalam Udhayakumar ${ }^{2}$, Peter D McElroy ${ }^{2,4}$, S Patrick Kachur ${ }^{2,3}$ and Naomi W Lucchi ${ }^{2}$

\begin{abstract}
Background: The World Health Organization (WHO) recommends parasitologic confirmation of suspected malaria cases before treatment. Due to the limited availability of quality microscopy services, this recommendation has become scalable following increased use of antigen-detecting malaria rapid diagnostic tests (RDTs) in many malaria-endemic countries. This study was carried out to monitor quality of RDT performance in selected health facilities using two quality assurance (QA) methods: reference microscopy and detection of parasite DNA by real-time quantitative polymerase chain reaction (qPCR) on dried blood spots (DBS).
\end{abstract}

Methods: Blood samples for QA were collected from patients undergoing RDT for diagnostic confirmation of malaria during two to three consecutive days per month in 12 health facilities in rural Tanzania. Stained blood smears (BS) were first examined at the district hospitals (BS1) and then at a reference laboratory (BS2). Discordant BS1 and BS2 results prompted a third examination. Molecular analysis was carried out at the Ifakara Health Institute laboratory in Bagamoyo.

Results: Malaria RDTs had a higher positivity rate (6.5\%) than qPCR (4.2\%) or microscopy (2.9\% for BS1 and 2.5\% for BS2). Poor correlation was observed between RDT and BS results: BS1 $(K=0.5), B S 2(K=0.43)$ and $\mathrm{gPCR}(K=0.45)$, challenging the utility of these tests for RDT QA. In addition, many challenges related to qPCR processing were recorded and long delays in obtaining QA test results for both microscopy and qPCR.

Conclusions: Overall there was limited agreement among the three diagnostic approaches and neither microscopy nor qPCR appear to be good QA options for RDTs under field conditions.

Keywords: Rapid diagnostic test (RDT), Microscopy, qPCR, Quality assurance (QA), Routine malaria tests

\section{Background}

The 2012 World Malaria Report identified tremendous achievements toward reduction of the global malaria burden [1]. Success has mainly been attributed to increased coverage of malaria control interventions such as use of insecticide-treated mosquito bed-nets (ITNs), indoor residual spraying (IRS), and use of effective treatments with artemisinin-based combination therapy (ACT). In 2010, the

\footnotetext{
* Correspondence: imasanja@ihi.or.tz

${ }^{1}$ Health System Sciences, Ifakara Health Institute, PO Box 78373, Dar es

Salaam, Tanzania

Full list of author information is available at the end of the article
}

World Health Organization (WHO) recommended parasitologic confirmation of suspected malaria cases before initiating anti-malarial treatment [2] and many malaria endemic countries in sub-Saharan Africa have adopted this policy $[3,4]$. The scale-up of antigen-detecting malaria rapid diagnostic tests (RDTs) for Plasmodium species forms a vital part of the strategy to confirm malaria infection prior to treatment in resource-poor settings [5].

Laboratory confirmation of malaria is being achieved, in part, due to the increased use of RDTs. With increased testing, the effect of incorrect test results is becoming a challenge in clinical management of patients suspected to

\section{Ciomed Central}

(c) 2015 Masanja et al.; licensee BioMed Central. This is an Open Access article distributed under the terms of the Creative Commons Attribution License (http://creativecommons.org/licenses/by/4.0), which permits unrestricted use, distribution, and reproduction in any medium, provided the original work is properly credited. The Creative Commons Public Domain Dedication waiver (http://creativecommons.org/publicdomain/zero/1.0/) applies to the data made available in this article, unless otherwise stated. 
have malaria infection [6,7]. Initial field trials of histidine rich protein (HRP)-2 based RDTs demonstrated a sensitivity and specificity of over $90 \%$ for Plasmodium falciparum at parasite densities over 200 parasites/ $\mu \mathrm{L}$ [8], particularly in laboratory or controlled settings. However, later studies have demonstrated that RDT sensitivity varied greatly between health facilities in Tanzania (18.8\% to85.9\%), with data difficult to interpret due to poor microscopy slide quality from some facilities [9]. Likewise other studies have reported significant variations in RDT sensitivity and specificity [10-13] and particularly when RDTs are exposed to adverse conditions, such as higher temperature [14].

Among the earlier recommendations of WHO was to assess performance of RDTs through periodic comparison of RDT results to reference microscopy [15]. In this recommendation, each health facility using RDTs was expected to submit blood smears from twenty RDT positive and twenty RDT negative patients monthly for evaluation [15]; however, in the wake of changing malaria transmission patterns this may not be feasible in areas of low transmission, as they may not have 20 positive RDTs in a month. Additionally, challenges of obtaining good quality blood smear results from lower level health facilities for further assessment at reference laboratories have been reported [16-19]. A need for a practical quality assurance (QA) procedure for RDTs was apparent.

Tanzania's Ministry of Health and Social Welfare through the National Malaria Control Programme (NMCP) began to deploy RDTs in 2010 in selected regions as a way to expand and strengthen malaria diagnostic capacity throughout the country. The NMCP recognized this effort to deploy RDTs would require a suitable QA method that could be adopted nationwide. The Ifakara Health Institute (IHI) in collaboration with the US Centers for Disease Control and Prevention (CDC) Malaria Branch and the U.S. President's Malaria Initiative (PMI), undertook a study in early 2010 to assess two methods of RDT QA and their timeliness. This approach included comparison of RDT used in routine care of patients, to reference microscopy and to a real-time quantitative PCR (qPCR) assay.

\section{Methods}

Tanzania adopted a phased approach to the scale-up of RDTs and first introduced the tests in three regions: Iringa (low endemicity), Kagera (high endemicity) and Pwani (high endemicity) in 2009. All levels of healthcare facilities (hospital, health center, and dispensary) were targeted to receive RDTs. These three regions accounted for approximately $12.8 \%$ of the Tanzania mainland's estimated 41.9 million population in 2010 [20].

\section{Study locations}

A convenience sample of 12 health facilities with high utilization rates in Iringa Region were selected to participate in this study; six in Mufindi District (Mafinga District Hospital, Kibao Health Center, Usokami Health Center, Malangali Health Center, Igomaa Dispensary and Sadani Dispensary) and six in Iringa Rural District (Tosamaganga Designated District Hospital, Idodi Health Center, Kimande Health Center, Mlowa Dispensary, Ifunda Dispensary and Ilambilole Dispensary). The selection was intended to include facilities in all levels of care: primary (dispensary), secondary (health center) and referral (district hospital).

\section{Sample collection}

RDT services were available for routine clinical care to all study facilities. To perform a test, health worker obtained finger-prick capillary blood specimens from patients suspected to have acute malaria infection. The blood was collected in a transfer device (either a capillary tube or loop) and placed in the appropriate well on the RDT where it is absorbed by the nitrocellulose paper. From the same finger prick an additional 2-3 drops of blood for a thick blood smear (BS) and 2-4 drops of blood for a dried blood spot (DBS) were collected. QA study samples were collected for a two to three day period each month, during the 5 months of data collection.

\section{Training}

Each facility received training on how to appropriately collect, label and store specimens. Health workers at all participating facilities were trained to perform RDTs by the local district council health management team (CHMT) during the national RDT roll out in 2009. In addition, all technicians at each district hospital laboratory had recently undergone a comprehensive microscopy competency training at the National Institute for Medical Research headquarters, Dar es Salaam, under the Walter Reed Army Institute of Research programme [21].

The RDTs used in this evaluation were those available at the government facilities through the Ministry of Health and Social Welfare and included SD Bioline ${ }^{\bullet}$, ParaHIT ${ }^{\bullet}$ and Paracheck ${ }^{\circ}$ obtained through the existing national procurement and distribution system. Log books and unique ID stickers were provided to record RDT results and for specimen tracking.

\section{Supervision, specimen processing, and QA logistics}

Study facilities received monthly visits from the district QA supervisor (a laboratory technician). Once a month, two days prior to the arrival of the supervisor, thick BS and DBS were collected from all suspected malaria patients receiving routine RDT at the health facility. A sticker was placed in the log book where RDT results were recorded. At the facility, the QA supervisor collected $40 \mathrm{BS} / \mathrm{DBS}$ pairs prepared in the previous two days, selecting pairs at random if greater than 40 were available. BS samples were sent directly to the district laboratory technician, who was blinded to RDT 
results, to be stained and examined (BS1). At the district laboratory, each BS was stained using 10\% Giemsa solution buffered to $\mathrm{pH} 7.2$, and stained for $8-15$ minutes. When a dilution of $5 \%$ was used, staining was done for 30 minutes.

All BS together with the corresponding DBS were sent to the IHI Bagamoyo laboratory for examination by a second microscopist (BS2), blinded to RDT and BS1 results. For both BS1 and BS2, a blood smear was declared negative after reading 100 high power fields. Parasites were counted in reference to 200 white blood cells. Any discordant results between BS1 and BS2 were resolved by a third microscopist (BS3), a senior technician from the Muhimbili University of Health and Allied Sciences (MUHAS).

\section{qPCR assays}

DBS samples were processed and analysed using qPCR in the IHI's research laboratory located in Bagamoyo, Pwani (qPCR1). As a validation, a subset of samples was retested at CDC, Atlanta, GA (qPCR2). DNA was extracted from DBS samples using a QIAamp DNA Mini Kit (QIAGEN, Valencia, CA (Qiagen method)). The DNA was aliquoted and stored at $-20^{\circ} \mathrm{C}$ until used. For quantification, a 10fold serial dilution of $P$. falciparum (3D7) was prepared at the Malaria Branch laboratory, CDC, Atlanta, GA and sent to IHI, Bagamoyo. Briefly, parasites were cultured on an orbital shaker to facilitate one parasite per red blood cell infection. The culture was synchronized by the sorbitol method to select for ring stage parasites which can reproducibly be used for quantification of DNA. The parasite density in parasites $/ \mu \mathrm{L}$ was determined. Ten-fold dilutions of the parasite culture were prepared with parasite densities ranging from 200,000 parasites $/ \mu \mathrm{L}$ to 20 parasites $/ \mu \mathrm{L}$. DNA was extracted from each dilution using the QIAamp DNA Mini Kit. All qPCR assays were performed using commercially available PCR buffer and taq polymerase (New England Biolabs, Ipswich, MA). Primers and a HEXlabeled Taqman probe to the P. falciparum beta-tubulin gene were designed and used as previously described [22]. DNA amplification was carried out following the cycling conditions: $95^{\circ} \mathrm{C}$ for 5 minutes, followed by 50 cycles of $95^{\circ} \mathrm{C}$ for 20 seconds and $58^{\circ} \mathrm{C}$ for 1 minute using the Mx3005p real-time PCR machine (Agilent technologies). The serially diluted 3D7 DNA standard was run in each experiment and was used to estimate the parasite density in the field samples. All samples were tested in triplicate. A thousand DNA samples were sent to CDC from which about 300 samples were systematically selected by picking every 3rd sample. These were processed by qPCR as described above and results used to validate the qPCR1 results from Bagamoyo.

\section{Data processing and analysis}

Data were entered in Microsoft Access (Microsoft Corporation, Microsoft Way- Redmond, Washington) and analyzed in STATA 11 (StataCorp, College Station, Texas). Only samples with a parasite density of 200 parasites $/ \mu \mathrm{L}$ as determined by $\mathrm{qPCR} 1$, were considered as positives in the comparison analysis, as this is considered the potential limit of detection for RDTs [23]. Samples with negative qPCR1 results were included as negatives and samples with lowdensity infection $(<200$ parasites $/ \mu \mathrm{L})$ by qPCR1 were excluded from analysis. Proportions of positive tests were compared across the two districts and against the three diagnostic methods using a chi-square test. Parasite densities from microscopy readings (BS1 and BS2, or BS1 and BS2/ BS3) were averaged to get mean variable for microscopy results (here referred to as BSsum). Chi-squared test assessed differences in proportion of positive tests by district. Cross tabulations between tests results were used to estimate each method's sensitivity, specificity, positive and negative predictive values for RDT, taking BSsum and qPCR1 as gold standard. McNemar's test was used to assess the difference in proportion of positive results by type of test. Kappa statistic was used to quantify the strength of the tests' results agreement.

\section{Ethical clearance}

The study protocol was determined to be exempt from ethical review by CDC and received IHI ethical approval number IHI/IRB/No. A 73-2009 as well as a national ethical permit from the Tanzanian National Institute for Medical Research: NIMR/HQ/R.8a/Vol. IX/1534. The sample transportation (import/ export) permit certificate No. 00007867, dated September 1st, 2010, was issued by the Tanzania Private Health Laboratories Board to allow movement of DNA aliquots to CDC laboratory in Atlanta, GA, USA.

Verbal agreements to use patients' level data for this analysis were acquired at the district health management level when permission was granted to conduct this study in the selected health facilities. No personal identifiers were used for patient's data.

\section{Results}

A total of 2,369 samples were collected from patients who attended participating health facilities and 2,324 (98\%) samples had complete information. During data entry, 487 (20.9\%) BS and/or DBS samples were found to have duplicate IDs due to a printing error of specimen labels. In addition, BS and DBS samples from 129 and 333 participants, respectively, were not collected which further reduced the number of available samples for microscopy and PCR analysis. Removal of these duplicates and missing samples left 1839 RDT samples, 1710 BS1, 1650 BS2 and 1506 DBS samples ready for analysis (Table 1). Sixty (3.5\%) slides with BS1 results could not be reviewed by the second reader (BS2) due to poor quality of smears and transportation challenges such as breakage. 
Table 1 Summary of all samples collected from 12 health facilities, in Iringa and Mufindi District Councils (DC)

\begin{tabular}{|c|c|c|c|c|c|c|}
\hline District & Name of Health facility & Expected samples & Total RDT & Total BS1 & Total BS2 & Total DBS \\
\hline Iringa DC & Idodi Health Center & 200 & 164 & 164 & 164 & 161 \\
\hline Iringa DC & Ifunda Dispensary & 200 & 196 & 195 & 196 & 156 \\
\hline Iringa DC & Ilambilole Dispensary & 200 & 185 & 180 & 170 & 156 \\
\hline Iringa DC & Kimande Health Center & 200 & 195 & 158 & 194 & 166 \\
\hline Iringa DC & Tosamaganga Hospital & 200 & 158 & 88 & 109 & 115 \\
\hline Iringa DC & Mlowa Dispensary & 200 & 160 & 156 & 156 & 158 \\
\hline Mufindi DC & Igomaa Dispensary* & 200 & 92 & 92 & 91 & 91 \\
\hline Mufindi DC & Kibao VA Health Center** & 200 & 154 & 154 & 154 & 76 \\
\hline Mufindi DC & Mafinga Hospital & 200 & 161 & 161 & 142 & 118 \\
\hline Mufindi DC & Malangali Health Center** & 200 & 152 & 140 & 55 & 101 \\
\hline Mufindi DC & Sadani Health Center* & 200 & 60 & 60 & 60 & 59 \\
\hline Mufindi DC & Usokami Health Center & 200 & 162 & 162 & 159 & 149 \\
\hline Total & & 2400 & 1839 & 1710 & 1650 & $1506^{* * *}$ \\
\hline
\end{tabular}

* Some samples had identical unique ID's, hence removed from the analysis.

** Some samples were not sent for reference reading and/ or further analysis in Dar es Salaam.

*** DBS were not available from every sample.

Of the 1,839 samples tested by RDT, 120 (6.5\%) were positive (Table 2). The local district microscopy (BS1) found 50 out of 1,710 (2.9\%) positives; reference microscopy (BS2) found 42 out of 1,650 (2.5\%) positives and qPCR1 found 64 out of 1,506 (4.2\%) positives (Table 2). Fifteen out of the 64 qPCR1 positive samples had a parasite density of $<200$ parasites $/ \mu \mathrm{L}$ and were not included in further comparative analysis with RDT and BS results. Malaria RDT had a higher overall positivity rate than microscopy and qPCR1, even when stratified by district (Table 2). The prevalence of malaria was found to be higher in Iringa District by both microscopy (BSsum) and qPCR1, but not by RDT which demonstrated higher positivity in Mufindi District (Table 2).

Overall, the sensitivity of RDTs was $85.3 \%$ and $65.3 \%$ with microscopy (BSsum) and qPCR1 used as the gold standard, respectively. The predictive value positive of RDT was very low by both microscopy (BSsum, 31.5\%) and qPCR1 (33.3\%) (Table 3). RDT results were poorly correlated with both microscopy BS1 $(\mathrm{K}=0.50)$, BS2 ( $\mathrm{K}=$ 0.43 ) and qPCR1 $(K=0.45)$ results (Table 4$)$. Microscopy results between the local district (BS1) and reference laboratories (BS2) showed 98.6\% concordance (McNemar test) and a high inter-observer agreement $(\mathrm{K}=0.75$, $\mathrm{p}=0.05$ ) between them. Of the 300 DNA samples processed by qPCR2, 270 samples had satisfactory results. The qPCR1 and qPCR2 results had $88.2 \%$ concordance, but were poorly correlated $(\mathrm{K}=0.32, \mathrm{p}<0.01)$.

Processing of BS1 took an average of 8 days (range $=1-$ 30 days), while that of BS2 averaged 36 days (range = 16-90 days). Significant delays (sometimes up to months) were observed in processing and obtaining qPCR1 results, due to technical challenges including equipment breakdown, lack of in-country expertise to repair the apparatus necessitating shipment to South Africa and short shelf-life of reagents.

\section{Discussion}

The aim of this study was to determine which of two methods, local qPCR (qPCR1) or microscopy (BS1), would provide optimal QA for RDTs in routine care. Neither of the two QA methods investigated correlated very well with RDT results, implying that these two tests (as performed

Table 2 Number tested and number positive according to diagnostic test performed on specimens obtained from patients who attended study facilities - Iringa and Mufindi District Councils (DC)

\begin{tabular}{|c|c|c|c|c|}
\hline \multirow{2}{*}{$\begin{array}{l}\text { Diagnostic } \\
\text { test }\end{array}$} & \multirow{2}{*}{$\frac{\text { Total }}{\text { Tested Positive (\%) }}$} & \multirow{2}{*}{$\frac{\text { Iringa DC }}{\text { Tested Positive (\%) }}$} & \multirow{2}{*}{$\frac{\text { Mufindi DC }}{\text { Tested Positive (\%) }}$} & \multirow[b]{2}{*}{ P value* } \\
\hline & & & & \\
\hline RDT & 1839120 (6.5) & $109262(5.7)$ & $74758(7.7)$ & 0.07 \\
\hline $\mathrm{BS} 1$ & $171050(2.9)$ & 94134 (3.6) & $76916(2.1)$ & 0.11 \\
\hline BS2 & $165042(2.5)$ & 98933 (3.3) & $6619(1.4)$ & 0.025 \\
\hline BS-sum & 155534 (2.2) & 93329 (3.1) & $6225(0.8)$ & 0.002 \\
\hline qPCR1 & $150664(4.2)$ & $91255(6.0)$ & 5949 (1.5) & $<0.001$ \\
\hline
\end{tabular}

${ }^{*}$ Chi-square test comparing district-level results. 
Table 3 RDT sensitivity, specificity and predictive values* reference standard test; $P P V=$ positive predictive value; NPP = negative predictive value

\begin{tabular}{lllll}
\hline Test combination (n) & Sensitivity & Specificity & PPV & NPV \\
\hline RDT/ BS-sum* (1555) & $85.3 \%$ & $95.8 \%$ & $31.5 \%$ & $99.7 \%$ \\
RDT/ qPCR1* (1506) & $65.3 \%$ & $95.6 \%$ & $33.3 \%$ & $98.8 \%$ \\
\hline
\end{tabular}

*Reference standard test; $\mathrm{PPV}=$ positive predictive value; $\mathrm{NPP}=$ negative predictive value.

here) may not be ideal for RDT QA or that inherent limitations of RDTs and/or of the RDT performer may cause inaccurate RDT test results. For example, RDTs are known to detect persistent HRP-2 antigen even after the successful treatment of malaria and clearance of asexual parasites $[8,24,25]$. The presence of circulating antinuclear antibodies such as in patients with rheumatoid arthritis, although a rare occurrence, has also been shown to give false positive RDT results $[24,26]$. In addition, studies in other malaria endemic areas have associated false positivity of RDTs with presence of gametocytes in blood [27] and acute schistosomiasis [28]. These other issues were not investigate in this study, but the higher positivity observed with RDT as compared to the other two tests can be partly explained by the detection of persisting HRP-2 antigen.

Other factors known to lead to false positivity by RDTs include inappropriate testing procedures (e.g., substituting test kit buffer solution with other liquids such as normal saline, distilled water, tap water or buffer from different kits/lots/ batches) [29]. Incorrect reading of RDT results by health workers during RDT testing is also known to influence test performance $[3,30]$. It is highly likely that health worker performance played a role in our findings because there is no other reason for RDT positivity rates to be higher in Mufindi district when all other malaria test results (BS1, BS2, qPCR1 and qPCR2) were lower in this district. Furthermore, community based surveys (through the Tanzania HIV and Malaria Indicator Surveys) and health facility data (as reported by District Health Information System 2) support higher transmission in Iringa district council than Mufindi district [31]. Although assessment of testing procedure during sample collection and supervisory visits showed very good adherence to the testing procedures it is also prone to observation bias (Hawthorne effect), hence a need to emphasize continued supportive supervision from health managers. False negative RDT results due to prozone effect have been reported [32]; however, there were no observed correlation between very high parasite density as detected by qPCR1 and microscopy with negative RDT results.

Studies have shown that the sensitivity of RDT compared to PCR and microscopy decline sharply at low parasite densities [9,33], therefore, care must be taken when comparing RDT results with molecular based diagnostic methods. The qPCR assay used in this study was selected on the basis that we would be able to quantify parasite densities and limit the comparison to RDT expected threshold of detection [8]. In this study, 15 qPCR1 positive samples had parasitemia of less than 200 parasites $/ \mu \mathrm{L}$ and only two of these were detected as positive by RDT. Despite excluding these samples, we did not observe good correlation between RDT and qPCR1 unlike previous studies where high agreement between RDT and PCR [33-35] was reported.

Prompt responsiveness to problems with the quality of RDTs will ensure timely remedial strategies such as recalling defective $\mathrm{RDT}$, providing refresher training to laboratory staff, or implementing closer supervision and follow-ups. This level of performance can only be practical where there is timely reporting of results to allow effective feed back into the system. Therefore, the timeliness of a QA test is an important consideration when selecting an appropriate QA test. The average microscopy processing time observed in this study was eight (8) days for district health facilities and 36 days at the reference laboratory. The lower end of this duration (i.e., 8 days) may be acceptable for use in routine QA, but improvement is needed to shorten this time to a fewer number of days.

The significant delays we observed in sample processing via $\mathrm{qPCR} 1$ and the fact that it was poorly correlated with qPCR2 suggest that this may not be a suitable method of QA in this setting. However, newer and simpler molecular assays that can overcome this limitation may provide good alternatives. This may include assays such as the loop mediated isothermal amplification (LAMP) assays [36] and Photo-induced Electron Transfer-PCR (PET-PCR) [37] that have shown to work in similar settings. The LAMP assay appears to be the most promising because of its simplicity, rapid turn around and sensitivity [38]. The potential of using

Table 4 Proportion of agreements between mRDT and microscopy and qPCR (McNemar comparison, Kappa statistic and correlation)

\begin{tabular}{llllll}
\hline Tests combination & Observed agreement & Expected agreement & McNemar p-value & Kappa statistic* & Correlation \\
\hline RDT- BS1 $(n=1710)$ & $95.6 \%$ & $91.2 \%$ & $<0.01$ & 0.50 & 0.54 \\
RDT - BS2 $(n=1650)$ & $95.2 \%$ & $91.5 \%$ & $<0.01$ & 0.43 & 0.48 \\
RDT - qPCR1 $(n=1506)$ & $94.9 \%$ & $90.7 \%$ & $<0.01$ & 0.45 & 0.47 \\
BS1 - BS2 $(n=1508)$ & $98.6 \%$ & $94.5 \%$ & $=0.05$ & 0.75 & 0.76 \\
\hline
\end{tabular}

Kappa statistic*: $p<0.01$. 
used RDT devices as a source of parasite DNA for molecular analysis to monitor RDT performance in the field [39] should be further explored in different settings, keeping in mind the differences in detection limits of the two tests.

The importance of in-service training was clear in this study. All technicians were trained in microscopy before the study and the level of technical competence shown by the districts' microscopists in this study, to stain and read slides, was encouraging. A good agreement between health facility and reference laboratory microscopy results was observed. This was a great improvement compared to previous result which demonstrated lack of competence in health facility microscopy [9]. The fact that microscopy is technically less challenging than qPCR and that the performance shown by the districts' technicians was better correlated with the reference microscopists after training, demonstrates the practicality of using microscopy-based QA for monitoring performance of RDTs in routine care as previously indicated [40]. However, the lack of agreement with RDT in this study in addition to the long delays observed in obtaining microscopy results brings to question the use of microscope as a QA test for RDTs.

The new WHO guidelines for quality control of RDT in field use are focused on quarterly supportive supervision of RDT performers with assessment of the testing procedure in clinical settings [39]. This intense level of quality control at health facility level requires dedicated teams of supervisors who will visit the health facilities and perform the necessary assessment and provide the appropriate corrective action to address noted deficiencies. Poor supervision and adherence to procedures may result in poor quality RDT results, as previously reported in other parts of Tanzania [41]. The use of positive control wells which consist of lyophilized recombinant proteins (mainly HRP2) which can be reconstituted in the field and used to test the correct performance of RDTs has been recommended [42]. An alternative to this is the proposed use of wellstandardized P. falciparum - infected dried blood samples in a tube that can also be used as positive control samples for monitoring RDT performance [43].

\section{Conclusions}

As highlighted in this study, finding an appropriate QA strategy for RDTs is challenging given the many possible factors that can compromise results from both the RDT and the selected QA test. Ensuring performer supervision in combination with the use of positive control samples to ascertain RDT performance is likely to provide the most practical and measureable QA approach for RDTs.

\section{Competing interests}

The authors declare that they have no competing interests.

\section{Authors' contributions}

MIM: data collection, data analysis and preparation of first draft. MLM: study design, data analysis revision of draft manuscript. MM, DS: sample analysis, revision of manuscript. VK, PM, SPK: study design, revision of manuscript. NL: study design, sample analysis, data analysis and revision of manuscript. All authors have read and approved the final version of this work.

\section{Acknowledgements}

This work was made possible by the generous support from the American people (USAID) and the US President's Malaria Initiative. Conclusions reached in this report are those made by the investigators (authors) and do not reflect views of the USAID, PMI or the US Government, or their Institutions. The authors would like to acknowledge immense support and contribution made by the National Malaria Control programme of the Tanzanian Ministry of Health and Social Welfare (case management cell); District Medical Officers and the Council Health Management Teams of Iringa and Mufindi District Councils; Clinician in-charge and health workers in 12 participating health facilities; IHI drivers; field workers for health workers' qualitative enquiry; US President's Malaria Initiative- Tanzania Office: $I H I$ administration, data unit and procurement unit(s), laboratory technician of Iringa Regional Hospital, Iringa District Council laboratory supervisor and Mufindi laboratory technicians at Mafinga Hospital.

\section{Author details}

${ }^{1}$ Health System Sciences, Ifakara Health Institute, PO Box 78373, Dar es Salaam, Tanzania. ${ }^{2}$ Malaria Branch, Centers for Global Health, US Centers for Disease Control and Prevention, Atlanta, GA, USA. ${ }^{3}$ US Public Health Services, Rockville, Maryland, USA. ${ }^{4}$ US President Malaria Initiative (PMI), Dar es Salaam, United Republic of Tanzania.

Received: 20 October 2014 Accepted: 30 January 2015

Published online: 19 February 2015

\section{References}

1. WHO. World Malaria Report 2012. Geneva: World Health Organization; 2012.

2. WHO. Guidelines for the treatment of malaria. 2nd ed. Geneva: World Health Organization; 2010.

3. Harvey SA, Jennings $L$, Chinyama M, Masaninga F, Mulholland K, Bell DR. Improving community health worker use of malaria rapid diagnostic tests in Zambia: package instructions, job aid and job aid-plus-training. Malar J. 2008;7:160

4. Zurovac D, Njogu J, Akhwale W, Hamer DH, Larson BA, Snow RW. Effects of revised diagnostic recommendations on malaria treatment practices across age groups in Kenya. Trop Med Int Health. 2008;13:784-7.

5. WHO/TDR/FIND. Malaria Rapid Diagnostic Test Performance. Summary results of WHO Malaria RDT Product Testing: Rounds 1-3 (2008-2011). Geneva, Switzerland: World Health Organization; 2011.

6. Nankabirwa J, Zurovac D, Njogu JN, Rwakimari JB, Counihan H, Snow RW, et al. Malaria misdiagnosis in Uganda - implications for policy change. Malar J. 2009:8:66

7. Allen LK, Hatfield JM, DeVetten G, Ho JC, Manyama M. Reducing malaria misdiagnosis: the importance of correctly interpreting Paracheck Pf "faint test bands" in a low transmission area of Tanzania. BMC Infect Dis. 2011;11:308.

8. Moody A. Rapid diagnostic tests for malaria parasites. Clin Microbiol Rev. 2002;15:66-78.

9. McMorrow ML, Masanja MI, Abdulla SM, Kahigwa E, Kachur SP. Challenges in routine implementation and quality control of rapid diagnostic tests for malaria-Rufiji District, Tanzania. Am J Trop Med Hyg. 2008;79:385-90.

10. Mason DP, Kawamoto F, Lin K, Laoboonchai A, Wongsrichanalai C. A comparison of two rapid field immunochromatographic tests to expert microscopy in the diagnosis of malaria. Acta Trop. 2002;82:51-9.

11. Reyburn H, Mbakilwa H, Mwangi R, Mwerinde O, Olomi R, Drakeley C, et al Rapid diagnostic tests compared with malaria microscopy for guiding outpatient treatment of febrile illness in Tanzania: randomised trial. BMJ. 2007:334:403.

12. Mueller I, Betuela I, Ginny M, Reeder JC, Genton B. The sensitivity of the OptiMAL rapid diagnostic test to the presence of Plasmodium falciparum gametocytes compromises its ability to monitor treatment outcomes in an area of Papua New Guinea in which malaria is endemic. J Clin Microbiol. 2007;45:545-50. 
13. Laurent A, Schellenberg J, Shirima K, Ketende SC, Alonso PL, Mshinda H, et al. Performance of HRP-2 based rapid diagnostic test for malaria and its variation with age in an area of intense malaria transmission in southern Tanzania. Malar J. 2010;9:294.

14. Chiodini PL, Bowers K, Jorgensen P, Barnwell JW, Grady KK, Luchavez J, et al. The heat stability of Plasmodium lactate dehydrogenase-based and histidine-rich protein 2-based malaria rapid diagnostic tests. Trans R Soc Trop Med Hyg. 2007;101:331-7.

15. WHO. Update on $\mathrm{WHO}$ procedures for selection and use of quality malaria RDTs. Geneva, Switzerland: World Health Organization; 2008.

16. Kachur SP, Nicolas E, Jean-François V, Benitez A, Bloland PB, Jean YS, et al. Prevalence of malaria parasitemia and accuracy of microscopic diagnosis in Haiti, October 1995. Pan Am J Public Health. 1998:3:35-9.

17. Dowling MAC, Shute GT. A comparative study of thick and thin blood films in the diagnosis of scanty malaria parasitaemia. Bull World Health Organ. 1966;34:249-67.

18. Bejon P, Andrews L, Hunt-Cooke A, Sanderson F, Gilbert SC, Hill AVS. Thick blood film examination for Plasmodium falciparum malaria has reduced sensitivity and underestimates parasite density. Malar J. 2006:5:104

19. Barat L, Chipipa J, Kolczac M, Sukwa T. Does the availability of blood slide microscopy for malaria at health centers improve the management of persons with fever in Zambia? Am J Trop Med Hyg. 1999;60:1024-103.

20. NBS. Tanzania in figures 2010. Dar es Salaam, Tanzania: National Bureau if Statistics, Ministry of Finance, United Republic of Tanzania; 2011.

21. Ohrt C, Obare P, Nanakorn A, Adhiambo C, Awuondo K, O'Meara WP, et al. Establishing a malaria diagnostics centre of excellence in Kisumu, Kenya. Malar J. 2007:6:79

22. Price RN, Uhlemann AC, Brockman A, McGready R, Ashley E, Phaipun L, et al. Mefloquine resistance in Plasmodium falciparum and increased pfmdr1 gene copy number. Lancet. 2004;364:438-47.

23. WHO/TDR. Malaria rapid diagnostic test performance: results of WHO product testing of malaria RDTs: round 1. Geneva, Switzerland: World Health Organization/TDR/FIND; 2008

24. Grobusch MP, Jelinek T, Hanscheid T. False positivity of rapid antigen detection tests for diagnosis of Plasmodium falciparum malaria: Issue appears to be more complicated than presented. J Clin Microbiol. 1999;37:3781-2.

25. Mayxay M, Pukrittayakamee S, Chotivanich K, Looareesuwan S, White NJ. Persistence of Plasmodium falciparum HRP-2 in successfully treated acute falciparum malaria. Trans R Soc Trop Med Hyg. 2001;95:179-82.

26. Grobusch MP, Alpermann U, Schwenke S, Jelinek T, Warhust DC. Falsepositive tests for malaria in patients with rheumatoid factor. Lancet. 1999:353:297.

27. Tjitra E, Suprianto S, McBroom J, Currie BJ, Anstey NM. Persistent ICT malaria P.f/P.v panmalarial and HRP2 antigen reactivity after treatment of Plasmodium falciparum malaria is associated with gametocytemia and results in false-positive diagnoses of Plasmodium vivax in convalescence. J Clin Microbiol. 2001;39:1025-31.

28. Leshem E, Keller N, Guthman D, Grossman T, Solomon M, Marva E, et al. Falsepositive Plasmodium falciparum histidine-rich protein 2 immunocapture assay results for acute schistosomiasis caused by Schistosoma mekongi. J Clin Microbiol. 2011:49:2331-2.

29. Gillet $\mathrm{P}$, Mori M, Van den Ende J, Jacobs J. Buffer substitution in malaria rapid diagnostic tests causes false-positive results. Malar J. 2010;9:215.

30. Rennie W, Phetsouvanh R, Lupisan S, Vanisaveth V, Hongvanthong B, Phompida S, et al. Minimising human error in malaria rapid diagnosis: clarity of written instructions and health worker performance. Trans R Soc Trop Med Hyg. 2001;101:9-18.

31. District Health Information 2 (DHIS 2) [http://dhis.moh.go.tz/dhis-web-pivot/ app/index.html]

32. Gillet P, Scheirlinck A, Stokx J, De Weggheleire A, Chauque HS, Canhanga ODJV, et al. Prozone in malaria rapid diagnostic tests: How many cases are missed? Malar J. 2011;10:166.

33. Farcas GA, Zhong KJ, Lovegrove FE, Graham CM, Kain KC. Evaluation of the Binax NOW ${ }^{\circledR} I C T$ test versus polymerase chain reaction and microscopy for the detection of malaria in returned travellers. Am J Trop Med Hyg. 2003;69:589-92.

34. Pieroni $P$, Mills $C D$, Ohrt $C$, Harrington MA, Kain KC. Comparison of the ParaSight ${ }^{\mathrm{TM}}-\mathrm{F}$ test and the ICT Malaria $\mathrm{Pf}^{\mathrm{TM}}$ test with the polymerase chain reaction for the diagnosis of Plasmodium falciparum malaria in travellers. Trans R Soc Trop Med Hyg. 1998;92:166-9.
35. Nicastri E, Bevilacqua N, Schepisi MS, Paglia MG, Meschi S, Ame SM, et al. Accuracy of malaria diagnosis by microscopy, rapid diagnostic test, and PCR methods and evidence of antimalarial overprescription in non-severe febrile patients in two Tanzanian hospitals. Am J Trop Med Hyg. 2009;80:712-7.

36. Lucchi NW, Demas A, Narayanan J, Sumari D, Kabanywanyi A, Kachur SP, et al. Real-time fluorescence loop mediated isothermal amplification for the diagnosis of malaria. PLoS One. 2010;5:e13733.

37. Talundzic E, Maganga M, Masanja IM, Peterson DS, Udhayakumar V, Lucchi NW. Field evaluation of the photo-induced electron transfer fluorogenic primers (PET) real-time PCR for the detection of Plasmodium falciparum in Tanzania. Malar J. 2014;13:31.

38. Hopkins H, Gonzalez IJ, Polley SD, Angutoko P, Ategeka J, Asiimwe C, et al. Highly sensitive detection of malaria parasitemia in a malaria-endemic setting: performance of a new loop-mediated isothermal amplification kit in a remote clinic in Uganda. J Infect Dis. 2013;208:645-52.

39. WHO. Universal access to malaria diagnostic testing: an operational manual. Geneva: World Health Organization; 2011.

40. Ishengoma DS, Lwitiho S, Madebe RA, Nyagonde N, Persson O, Vestergaard LS, et al. Using rapid diagnostic tests as source of malaria parasite DNA for molecular analyses in the era of declining malaria prevalence. Malar J. 2011;10:6.

41. Chandler Cl, Jones C, Boniface G, Juma K, Reyburn H, Whitty CJ. Guidelines and mindlines: why do clinical staff over-diagnose malaria in Tanzania? A qualitative study. Malar J. 2008;7:53.

42. WHO. Malaria Rapid Diagnostic tests: RDT Evaluation Programme- Positive Control Wells [http://www.wpro.who.int/malaria/sites/rdt/who_rdt_evaluation/control_wells.html]

43. Aidoo M, Patel JC, Barnwell JW. Dried Plasmodium falciparum-infected samples as positive controls for malaria rapid diagnostic tests. Malar J. 2012;11:239

\section{Submit your next manuscript to BioMed Central and take full advantage of:}

- Convenient online submission

- Thorough peer review

- No space constraints or color figure charges

- Immediate publication on acceptance

- Inclusion in PubMed, CAS, Scopus and Google Scholar

- Research which is freely available for redistribution 